\title{
Some reflections on renal care
}

Fernando Macário

SVP, Chief Medical Officer

Diaverum

\section{THE NEED TO OVERCOME UNEQUAL ACCESS TO RENAL CARE}

While we wait for a miracle drug to treat kidney diseases, we still have to live with the increased numbers of people in need of renal replacement therapy (RRT).

People with CKD still face the anxiety of one day starting dialysis or receiving a renal transplant, or, even more probably, the chance of a serious or even fatal cardiovascular event. When we consider the global picture, we must recognize that starting RRT is still only for those lucky enough to have access to renal care.

In many regions of the globe, there is still no or only limited access to renal care and to RRT. That is the sad reality; we simply do not have the means to provide minimal standards of care for millions of people. That is true for all stages of CKD and for access to RRT. Still today, in many countries, renal care dialysis is available to only a very few and, even so, with low standards, on an irregular basis and with poor access to fundamental medications to treat basic aspects such as anaemia, CKD-related bone mineral disease and other comorbidities. ${ }^{1,2}$

It is our responsibility as nephrologists to raise awareness through our scientific organizations of this unequal access to basic renal care. International health organizations have the same duty to lobby and providers of renal care must work with health authorities to find solutions of value that will permit minimal standards of care for these forgotten populations. To achieve this is a social responsibility we all must take on. New solutions are needed, such as strong support with digital solutions, and new methods such as automated algorithms of care pathways based on machine learning and artificial intelligence to bring acceptable care where specialized human resources are insufficient.

Digital solutions, supported by basic facilities and local staff, that connect renal professionals and patients in remote areas can improve care and bring expertise where we cannot dream of having specialized care in the near future. Digitalization will allow us to reach where health professionals are scarce and free them up for more differentiated tasks. Digitalization will remove physical barriers and bring care to more of those in need, even in remote or poorer areas. Virtual nurses or physicians will not abolish human decision, but a strong digital support will improve accuracy of diagnosis and treatment and communication. It is important to talk about the basic aspects of unequal access before we even think about other technical miracles of renal care we all want to see.

\section{NEW RESPONSIBILITIES FOR RENAL CARE PROVIDERS - SOME PERSPECTIVES}

We all dream of a point where dialysis and transplantation will no longer be needed. We are still not there; maybe not even any closer to it. Despite the promises we see every day in our social media that dialysis will end, that implanted devices to replace failing kidneys will be widely available tomorrow and that we are almost producing organs in the lab for transplantation, we still have to focus on how to treat the person with CKD we have in front of us. ${ }^{3}$ The most effective way of diminishing the burden of CKD for patients is focusing on the person in a holistic way. Beyond the need for compassion and dedication of highly specialized health professionals, there is a need for ensuring the most accurate diagnoses and to promote public health education about critical health issues. We must take into account psychological and social realities. Families and caregivers must be involved and patient education and health literacy promoted. This cannot be achieved without a multidisciplinary and integrated care approach. Nephrologists can only feel empowered if they divide the multiple dimensions of CKD care with other health care professionals. With this approach, they will be the maestros of a well-tuned orchestra playing a masterpiece and not the solo performers of an isolated piece of music. The isolated healthcare professional is something of the past - teamwork-coordinated approach is the way to go. Only by addressing the multiple aspects of chronic kidney disease in this way can we achieve the ultimate goals for the person with CKD - to live as long as possible without RRT and with a good quality of life. We need to understand patient needs and perception of care to arrive at a well-structured care plan.

This, of course, includes optimum use of available resources. The care providers and health professionals involved and also the health authorities and politics must all bear responsibility. Here we see many obstacles. In the search for quality in healthcare, we see health authorities creating legislations that are not enforced by many people because these legislations are absurd and outlandish, and we see regulators auditing the care pathway instead of auditing the clinical outcomes of the care provided, using a constructive framework. We need a different approach if we do not want to jeopardize the quality of care we have today.

While we wait for the miracles of CKD care, we still have a long way to go to optimize care today. The future will be built by evolution more than revolution. Despite the clinical excellence of the care currently provided by Portuguese health professionals, there is still room for a new clinical strategy toward value-based care. We 
need to develop a wide array of metrics of success, so that we know what good quality looks like, what good service feels like and what efficient care costs. While our assessment of quality today is mostly process metrics, we should try to move closer to outcome metrics in the future.

Modern medicine is targeting a new and exciting direction. In parallel with fantastic technical innovations, we must drive clinical care to new standards through high levels of standardization based on protocol-oriented intervention in critical areas of renal care, relying on evidence-based clinical and therapeutic protocols. It is fundamental to measure clinical performance and outcomes with comprehensive and standardized methods. Digitalization and analysis of data is of paramount importance for accompanying results of strategic plans and to audit compliance with their implementation. With today's data management, it is possible to learn from data. New information technology support will also free up clinical staff and improve communication with all players in the care pathway. We have an exciting new world ahead of us.

Patient-centred medicine focusing on the value of the interventions is changing the face of clinical care and this new face is more human. Integrated renal care through models of Patient Care Coordination (PCC) drives patient satisfaction, dovetails with a holistic approach and improves efficiency of care, avoiding duplication of interventions, uncoordinated care and added costs. Inclusion of the patient in the decision-making process through promotion of literacy in healthcare with patient educational programmes and, when appropriate, involving the family and caregivers in the PCC model can improve the efficiency of our interventions. Personalization of care can improve relevant outcomes; individualization of care may lead to increased efficiency. Holistic care of renal patients is more effective than the biomedical model of care in the treatment of chronic disease. $^{4}$

The pillars of intervention in renal care should include strategies aimed at improving standardization, diminishing unnecessary variability of care, providing the best possible care to the greatest number of patients with ethical cost containment. Chronic renal disease patients are very complex with many comorbidities and a complex psychological and sometimes social environment. The definition of the most relevant outcomes is of paramount importance to design the correct intervention in renal patients. The leading causes of morbidity in renal failure hemodialysis patients are cardiovascular disease, infectious diseases and complications of vascular access. Thirty to $40 \%$ of patients are diabetic. We also know that circa $30 \%$ of patients re-hospitalized within 30 days of discharge from a previous hospitalization will die. It does seem logical that programs optimizing control of these comorbidities will improve quality of life and most probably diminish mortality. Protocoled interventional plans and therapeutic protocols will drive standardization and optimization of care.

In order to improve value, value must be measured. While our assessment of quality today is focused primarily on clinical care processes, safety, and cost, we should try to move from process of care to outcome metrics in the future. Quality measurement programs must define the end goal, not the process for achieving it. In the meantime, if we want to ensure that performance indicators are fostering a value-based delivery system, renal care providers and authorities must cooperate to define what is relevant and evidence-based to measure. ${ }^{5}$

For the creation of value, ideally renal health care providers must be in the position of providing care through all pathways of care. In renal disease, this means intervention in prevention in all stages of renal disease and the possibility of providing all modalities of treatment. Care of renal patients before stage five CKD may decrease cardiovascular events and delay progression of kidney disease, eventually reducing the higher social and economic burden of end stage renal disease. Good predialysis care has an important impact on reducing mortality before and after starting renal replacement therapy and permits better individualization of care. Stage 5 CKD patients must be offered the choice of in-centre or home hemodialysis, peritoneal dialysis, palliative care and access to renal transplantation programs. $^{6}$

There is no future in medicine without strong research. Research must be supported; clinical research is a heavy responsibility of medical professionals, mainly of physicians and nurses. It is a corporate responsibility of any medical provider to support good research initiatives. It is also important to be well positioned to embrace future developments in renal medicine. New exciting drugs to halt or even reverse chronic renal disease are being studied and those who treat earlier phases of renal disease will have the chance to test and use them. Optimum treatment of conditions that lead to chronic renal disease, such as diabetes and hypertension, will play an important part in preventing renal disease. Exciting new treatment for diabetes such as optimum wearable biofeedback systems for insulin delivery (bio artificial pancreas), production of pancreas islets with bioengineering either with stem cells or with xenotransplantation are underway, promising a bright new future. Wearable artificial kidney systems with biological renal functions not achievable by current dialysis systems will also revolutionize renal replacement therapy. Miniaturization will revolutionize dialysis treatment. The same can be said of biologically engineered kidneys, where there is a huge investment by the best research labs in the world. Not a reality today, and perhaps not in the short-term, but it will surely be a reality one day.

With their feet on the ground, nephrology providers must be partners to healthcare authorities and people with chronic renal disease to improve access to renal care and innovation. The patient must be empowered and heard to make informed decisions about his own care.

Disclosure of potential conflicts of interest: none declared

\section{References}

1. Burden, access and disparities in kidney disease. Crews DC, Bello AK, Saadi G; World Kidney Day Steering Committee. Clin Kidney J. 2019 Feb 18;12(2):160-166. doi: 10.1093/ckj/sfy128. eCollection 2019 Apr. PMID: 3097639

2. The global burden of kidney disease and the sustainable development goals https://www.altmetric.com/details.php?domain=www.who.int\&citation_id=43111413 Valerie A Luyckx a, Marcello Tonelli b \& John W Stanifer c, Bulletin of the World Health Organization 2018;96:414-422D. doi: http://dx.doi.org/10.2471/BLT.17.206441 
3. Ambulatory Hemodialysis-Technology Landscape and Potential for Patient-Centered Treatment. Hojs N, Fissell WH, Roy S. Clin J Am Soc Nephrol. 2019 Nov 14. pii: CJN.01970219. doi: 10.2215/ CJN.01970219. [Epub ahead of print] Review. PMID: 31727617

4. Health care professionals' experiences and enactment of person-centered care at a multidisciplinary outpatient specialty clinic. Evén G, Spaak J, von Arbin M, Franzén-Dahlin Å, Stenfors T. J Multidiscip Healthc. 2019 Feb 14;12:137-148. doi: 10.2147/JMDH.S186388. eCollection 2019. PMID: 30858711

5. Development of an International Standard Set of Value-Based Outcome Measures for Patients With Chronic Kidney Disease: A Report of the International Consortium for Health Outcomes Measurement (ICHOM) CKD Working Group. Verberne WR, Das-Gupta Z, Allegretti AS, Bart HAJ, van Biesen W, García-García G, Gibbons E, Parra E, Hemmelder MH, Jager KJ, Ketteler M, Roberts C, Al Rohani M, Salt MJ, Stopper A, Terkivatan T, Tuttle KR, Yang CW, Wheeler DC, Bos WJW. Am J Kidney Dis. 2019 Mar;73(3):372-384. doi: 10.1053/j.ajkd.2018.10.007. Epub 2018 Dec 20. PMID: 30579710
6. Where Are You Going, Nephrology? Considerations on Models of Care in an Evolving Discipline. Piccoli GB, Breuer C, Cabiddu G, Testa A, Jadeau C, Brunori G. J Clin Med. 2018 Aug 3;7(8). pii: E199. doi: 10.3390/jcm7080199. PMID: 30081442

\section{Correspondence to:}

Fernando Macário

SVP, Chief Medical Officer, Diaverum

E-mail: fernando.macario@netcabo.pt 